\title{
EXPLORING THE DIVERSITY OF SERVICE WORLDS IN THE SERVICE ECONOMY
}

\author{
Michael Barrett \\ Judge Business School \\ University of Cambridge \\ Cambridge, U.K. \\ Elizabeth Davidson \\ Shidler College of Business \\ University of Hawaii at Manoa \\ Honolulu, HI U.S.A.
}

\section{INTRODUCTION}

The transformation of modern economies into predominantly service-based economies is happening on a global scale. While services are not new, the scale and complexity of globally dispersed services are growing rapidly. These transformations are enabled by - and often dependent on - information technologies and are fueled by processes of globalization. Transformational change provides opportunities for innovation in business models, collaborations, and work practices but also presents challenges to established practices within industries and organizations. The aims of the IFIP Working Group 8.2 Conference on IT in the Service Economy were to better understand the possibilities and challenges of these transformations and to examine key implications for organizations, their employees, and IT professionals in the $21^{\text {st }}$ century service economy.

\section{WHAT IS DRIVING THE TRANSFORMATION TO THE SERVICE ECONOMY?}

Growth in service sector employment and in the contribution from service sector industries to national economies has continued apace for some time. The shift from

Please use the following format when citing this chapter:

Barrett, M., and Davidson, E., 2008, in IFIP International Federation for Information Processing, Volume 267, Information Technology in the Service Economy: Challenges and Possibilities for the $21^{\text {st }}$ Century, eds. Barrett, M., Davidson, E., Middleton, C., and DeGross, J. (Boston: Springer), pp. 1-10. 
manufacturing-based to service-based economies began in the mid- $20^{\text {th }}$ century and is evident not only in highly developed economies but increasingly in developing nations. However, social, political, and economic trends are now interacting to amplify this shift (Bryson et al. 2004). For example, as per capita income rises and as individuals devote more time to work, demand for services, particularly personal and domestic services, increases. Demographic changes and increasing skills requirements for modern jobs have increased the demand for health and educational services. Most firms operate within extended production networks and employ sophisticated technologies in production processes; such networks require coordination services (such as accounting or legal services) and contribute to employment in professional service occupations. Requirements for interfirm coordination as well as outsourcing and externalization of service work (such as IT) contribute to the growth of specialized service providers (e.g., consulting firms). Governments influence service growth directly, as local, regional, national, and international governmental bodies extend their service offerings and payrolls, and indirectly through economic regulation, which stimulates growth in compliance-related services. International trade in services involves both provisioning of services remotely (e.g., with remote call centers) and relocation of clients to service areas (e.g., tourism).

When we consider these diverse factors, which contribute to growth in the level and variety of services in our modern economy, we begin to appreciate that the so-called service economy is much more than an economic shift; it is a transformation to a complex array of social, political, and economic service worlds. Bryson et al. (2004, p. 3) comment, "Service worlds are complex, evolving, heterogeneous, and long-established phenomena... a service world is also one in which there is a direct, even dialectical, relationship between service production and service consumption."

\section{INFORMATION TECHNOLOGIES AND SERVICE}

Given this broad view of the service economy, we might question what we mean by "service" and whether services in today's service worlds are essentially the same as services of the past. Of particular interest is how information technologies influence how we experience and how we innovate with services.

Services have been defined as intangible, heterogeneous, and perishable, and as requiring joint participation by the consumer and producer of the service using their skills and knowledge to produce (Gersung and Resengren 1973). Services have generally been viewed as more local and less subject to globalization than products. An example that illustrates this perspective is a consumer viewing a movie in a theater. The intangible service - entertainment - requires the consumer's cooperation to sit through the movie and presumably to enjoy it. If a particular seat in a theater at a particular showing goes unfilled, that instance of the service perishes (unlike the movie popcorn, which can await future consumption). Each patron at the showing may experience the service differently. Similarly, an organizational example of the traditional view of services is a public accounting firm auditing a client firm, utilizing the accountants' skills and knowledge and acting with the cooperation of the client firm, in order to satisfy regulatory requirements. The service is produced as the accountants' time is expended and thus is not "storable," although the artifacts of the service, such as the auditreport, are.

These traditional views of services are being challenged, particularly the differentiation between products and services. Gustafsson and Johnson (2003) suggest, for 
example, that "physical products are services waiting to happen." Vargo and Lusch $(2004,2008)$ similarly claim that all exchanges essentially are service exchanges, in which the customer and supplier co-create value with products or with services. Servitization, which generally involves adding value to products through services (often informational services) or offering products as services (e.g, software-as-a-service), further reduces the boundary between products and services.

This expanded understanding of the relationship between products and services can reveal opportunities for innovation and competitive advantage (Gustafsson and Johnson 2003). Moreover, embedding information and communication technologies in service offerings may alter other taken-for-granted characteristics of services. Using our movie example, "movies on demand" services available through home cable or the Internet enable the service provider to sell a viewing of the movie to consumers " 24 by 7 ." Does this service actually perish? Is co-presence necessary for co-creation of value? Although the service experience (entertainment) is intangible, a complex array of technological artifacts is required to deliver the service, and the quality of the artifacts undoubtedly influences the quality of the entertainment experience.

Thus, not only do products take on service characteristics through servitization, but with extensive use of IT in services, services may take on some of the characteristics typically associated with products, that is, standardization, storability, and separation of production and consumption of the service. Expanding our understanding of these complex interrelationships can, we suggest, point to a variety of opportunities for service innovation across a wide variety of socio-economic activities.

\section{GLOBALIZATION, IT, AND THE SERVICE ECONOMY}

Contrary to the traditional expectation that service production is essentially local, in fact, globalization of services is a widespread socio-economic trend in modern economies. Nearly 20 percent of international trade is trade in services. The IT industry and its professionals have been at the leading edge of the trend to globalize services, a trend that now includes many other information services such as legal, accounting, educational, health care, and other professional services.

Globalization has been the subject of much controversy because of the disruptions to local economies that can result. By globalization, we mean the geographic separation (yet increasing interconnection) of service providers and service consumers, whether individuals or firms, the movement of service workers or service consumers around the globe to deliver services, and the development of capabilities around the globe to provide services. Globalization undoubtedly provides new opportunities for service innovation, particularly in the knowledge-based and informational services, enabled by information and communication technologies (ICTs). At the same time, there are significant barriers such as language, cultural, regulatory, environmental, and time zone differences, which pose challenges to the provision of global services. Thus, when we consider IT and the transformations in the service economy, globalization and its effects become a very important focus of the research of the IFIP Working Group 8.2 community. 


\section{OVERVIEW OF THE BOOK}

The selection of completed research works, research-in-progress reports, panel discussions, and workshop papers presented at the 2008 IFIP Working Group 8.2 conference, "IT in the Service Economy," are documented in this volume. Collectively, these works address many of the themes outlined in our introduction: conceptualizing and theorizing about IT-enabled services, innovation in services through IT, growth of IT-enabled services in a broad array of service sectors, including government, healthcare, and other sectors, and outsourcing and globalization of IT services.

\subsection{Conceptualizing and Theorizing about IT-Enabled Services}

Recent developments in the services literature, particularly from the marketing and operations disciplines, portray an ongoing reflexiveness as to how services should best be conceptualized and theorized in today's service economy. However, there has been relatively little work in the IS discipline that has contributed to this growing debate. This is somewhat surprising since IT has not only played a significant role in aspects of service design and delivery, but calls into question previous conceptualizations of service, and suggests new conceptualizations (e.g., software as a service). It is therefore timely that the papers in this section take a step toward conceptualizing and theorizing ITenabled services. Five papers challenged different conceptualizations of IT-enabled service and service work and provided novel ways of theorization and conceptualization. Three of these papers examine primarily global, macro-level issues while the other two are focused more squarely at the organizational and work system level.

In challenging populist conceptualizations, Walsham is critical of the "flat world" rhetoric that suggests there are essentially no barriers to globalization of service work. While the inappropriateness of this caricature may be well recognized by many in the 8.2 community, Walsham argues that we need to raise up a critical voice that constructively challenges the notion of a relationship-free world and highlights the continued importance of cultural diversity and local specificity amongst other unevenness. He outlines critical themes for further research, namely identity and cross-cultural working, globalization, localization, and standardization, and the explicit focus on power, knowledge, and control in understanding global working in a non-flat world.

Continuing the theme of cultural diversity, Vorakulpipat and Rezgui provide an intriguing illustration of the importance of local and cultural particularities through their study in a Thai IT research service organization. They explore the influence of shyness as a socio-emotional characteristic of the Thai culture on knowledge management practices in this organization and the need to consider shyness barriers for understanding team working, structure and culture, training, knowledge sharing, and motivation for knowledge management in this cultural setting.

Addressing the ways in which government regulations promote the need for and development of information services, Butler, Emerson, and McGovern offer a novel and timely conceptualization of compliance-as-a-service and as a viable value proposition. Drawing on institutional theory, they examine the different pressures faced by an IT manufacturing organization and show that regulatory influences have developed the deepest response in terms of innovative compliance-oriented procedures and protocols. 
Alter challenges the service innovation literature for its predominant focus on largescale topics of globalization and eco-systems of interacting suppliers and consumers, and argues the need to address more micro levels. Specifically, he suggests that less attention has been given to a systems perspective and the development of specific localized systems. To address these shortcomings, he critically evaluates the existing definitions of service and service systems and argues for simpler, broader-based definitions. He also draws on three interrelated frameworks, the work system framework, the service value chain framework, and the work system life cycle model to conceptualize service innovation across a wide range of service systems.

Drawing on Deleuze and Guattari's theory development of rhizomes as nonhierarchical networks, Atkinson and Brooks offer a novel conceptualization of rhizomatic informatics to explore IT-enabled service work to reinterpret a classic study of a problematic ERP implementation in a major university. In so doing, they not only illuminate the conceptual richness of such an approach for understanding the nature and complexities of IT-enabled service work and practice but they also question Deleuze and Guattari's overarching focus of information systems as instruments of corporate arboreal control.

Two research-in-progress papers contribute to our understanding of socio-cognitive services inherent in distributed software teams in global service work and in theorizing the service encounter. First, Shen and Gallivan's study examines how distributed software teams can develop capabilities to perform successfully across temporal, geographic, and cultural boundaries. Their novel theoretical perspective extends and applies transactive memory systems and faultline models to examine inter-subgroup dynamics of these teams on knowledge coordination. Second, Ramiller and Chiasson seek to conceptualize and theorize the role of sense giving and sense making in the creation of ITenabled service encounters. They argue that in every service event one party's interpretations are being built upon the interpretations of others. They then develop a threelayer stratigraphy of sense-making and sense-giving in IT-enabled services to delineate the role of sense-making in the ongoing constitution of IT-enabled service events, the redesign of IT enabled service practices, and in the organizing vision discourse about the IT-enabled service.

\subsection{IT-Enabled Services in Industry Settings}

Traditional classifications of service industries have included retailing, wholesaling, transportation, financial services, healthcare, education, and so on. Classifying such diverse industries simply as service industries overlooks critical differences in the institutional environments that are likely to influence the ways in which information technologies are developed and applied, as well as the outcomes of IT use in these environments (Chiasson and Davidson 2005). The papers in this section address the specificity of industry context while drawing on and contributing to our knowledge of IT in service industries more generally.

Two papers examined the use of information technologies in healthcare settings, paying close attention to the array of institutional actors who participate in health IT systems. Andersen and Aanestad reported on the development and deployment of a community-wide support system for ambulatory psychiatric care for children in Norway. 
Drawing on the concept of generativity and information infrastructure, they found that the existing installed base could not encompass user-driven innovations, but that users were able to overcome resistance from infrastructure supporters to promote development of a new and more effective design. The intent and design of this system was consistent with the movement to home-based, self-care practices and patient empowerment in the healthcare industry. Vuokko and Karsten examined the work practices of nurses in a pediatric unit of a hospital prior to the introduction of electronic medical records. They suggested that actor-network theory and complexity theory provide appropriate analytic lenses to investigate how the varied interests of actors in this work setting influence the reordering of time-place arrangements and enactment of changes in coordination, communication, and information sharing practices with the new technology.

Continuing the research focus on work groups but in a different industry setting, Van Daalen Fuente, Chiasson, and Devadoss investigated the ways in which a face-to-face community of practice of stock traders engaged in knowledge exchange and community learning in a colocation setting, despite utilizing technologies designed for remote working. Feller, Finnegan, Fitzgerald, and Hayes studied interfirm exchanges in an open source service network and examined the social mechanisms that facilitate coordination and safeguarding of exchanges of the Zea Partners network. They found that restricting firms' access to the network, establishing a macro-culture within the network, collectively sanctioning member firms' unacceptable actions, and building the network's reputation were social mechanisms that enabled the network of firms to deliver commercial products to exploit peer produced software.

Two research-in-progress papers report initial findings on how new information technologies may affect work practices and industry norms. Loebecke, Huyskens, and Gogan presented initial findings of a study of RFID (radio frequency identification) tags in retailing. This case study outlined how consumers' experience in a retail setting may be improved through use of the technology and how mangers may more effectively meet customers' needs with the information available through RFID systems. Sawyer and Yi utilized macro-economic data to explore how information technologies may have influenced employment in and productivity of the real estate industry. Their preliminary analysis suggests that IT investments have contributed to value produced in this industry but have not resulted in work force reductions, despite open access to real estate data by customers.

\subsection{IT-Enabled Change in Public Services}

Examining the intersection of IT, service innovation, and growth in the service economy is particularly interesting in the public service sector. We would normally expect innovation and change to be difficult here, due to bureaucratic institutions and lack of market incentives. However, the research studies reported here emphasize that change is underway, as governments expand their regulatory roles into the domains of IT services and as they incorporate IT-enabled innovations to be more responsive to constituents.

Petrakaki's study of e-government initiatives in Greece illustrated the interaction of these issues. The opportunities for service innovation such as "one-stop shopping" often conflict with bureaucratic practices. Implementing so-called best practices and tech- 
nologies developed in commercial settings into the institutional environment of government may set in motion transformational changes in the nature of governmental service work; such changes are not uncontested by established interests and governmental actors and may lead to power shifts in the bureaucracy.

Tapia and Ortiz's study of municipal wireless network policy making processes explored how governmental services are expanding to encompass essential IT infrastructure services. They noted that unlike private enterprises, decision-making by governmental bodies necessarily involves public policy making, typically a top-down process. In the case of wireless broadband network services, a hotly contested area, local governments are jumping into the market, and the majority of policy making is now happening at the local level.

Three research-in-progress papers also considered themes of IT-enabled service innovation manifest in the actions and policies of governmental agencies. Maldonado and Tapia's assessment of Venezuela's policy for open source software adoption illustrates both the expansion of government regulation into the domain of IT and the growth in government services related to IT-in this case, promoting development of open source services and capabilities in the economy. Constantinides and Blackler's study of the National Program for IT in the United Kingdom similarly investigated the expansion of governmental services and oversight into IT activities. By examining the discursive activities of participants in a public review of this massive public healthcare IT project, the paper considered how attributions of success and failure are constructed and how coorientation may be negotiated across diverse interest groups through discourse. Bernardi's study of the introduction of IT-enabled change in public health services in Kenya adopted a micro-level view of institutional change to examine how formal policy structures are interpreted and enacted in micro-level actions in this setting.

\subsection{Outsourcing and Globalization of IT Services}

Outsourcing and offshoring have been a key feature of the IT services landscape over the past decade. While globalization of services facilitated by ICTs continues apace, there is a need for service work to meet the many social, cultural, and political challenges that influence their successful growth and development. The papers in this section highlight key issues inherent in the unevenness of global service work, the importance of respecting cultural diversity, and the ongoing challenge of managing knowledge processes. These papers cover both IT and business process outsourcing, and highlight a number of conceptual themes including legitimacy and trust, power and self-knowledge, boundary objects, and knowledge gaps.

Barrett, Hinings, and Oborn explored the dynamics of the offshoring relationship between a multinational firm and its Indian vendors in the provision of global software development. They highlighted the value of legitimacy management in unearthing the strategies and activities in gaining, maintaining, and repairing legitimacy at different points in the evolution of the relationship, and discussed the role of trust in managing legitimacy.

Four research-in-progress reports explored a range of issues and themes on IT and business process outsourcing. Komporozos-Athanasiou proposes an alternative focus to relationship management in understanding the high failure rates in global outsourcing 
arrangements. He suggests a focus on self-knowledge in client-focused maturity maturity assessment to realize potential outsourcing benefits and draws on coevolutionary theory to better understand the asymmetry of knowledge and power dynamics. Gregory and Prifling focused on ways in which IS service providers can leverage their client relationships over multiple projects at multiple levels for expertise development and knowledge integration. At the more operational level, Aman and Nicholson developed a theoretical framework to examine the knowledge gaps in offshore software development work when teams are separated by time, distance, and culture. The framework emphasizes the different types of knowledge held by team members in analyzing the potential for knowledge gaps. Finally, in examining business process offshoring around call center service work, Devadoss and Chiasson examined and complicated the typical utopian and dystopian views of automation. The utopian view portrays workers as having pure and unrestricted agency to pursue a knowledge-based career, while the dystopian view is that of an "electronic panopticon," with restricted and imprisoned individuals having few options. In reality, the picture is much more complex with human and nonhuman participants being linked by an underlying complexity of boundary work and boundary objects in what they view as a stable industry.

\subsection{Panels}

Four panel presentations engage in the debates highlighted in paper presentations about the nature of service and the possibilities for IT-enabled service innovation. In our first panel, Ramiller, Davidson, Wagner, and Sawyer take issue with the predominant focus on servitization of products in the discourse on the service economy and suggest that services are also being transformed into something akin to products - structured, standardized, scalable artifacts that separate the service provider and service consumer in the co-creation process. Each panelist explores the contradictory implications of servicitization and productization in an industry setting and the tensions this engenders. In the second panel, Alter, Gal, Lipien, Lyytinen, and Russo examine the implications of servitization for the structures, processes, and competencies of service provider and service consumer organizations and the role of IT in such changes. The panelists focus on new types of collaborative relationships in service value chains and question how collaborative relationships affect organizational identity, goals, and missions. The panel considers practical approaches to sorting out the implications of servitization for system and organizational design.

Feller, Finnegan, Lundell, and Nilsson continue this discussion by addressing servitization of peer production networks, and using the example of open source software consider how profitable service offerings might develop around the process of open source software production. Each panelist brings their considerable experience in open source software development to bear as they consider how services are developing in this domain, and what we might learn more generally about servitization in peer production networks.

Our fourth panel delves into e-health initiatives to consider how IT-enabled innovation in health service delivery provides new opportunities for the inclusion of patients and the community into health care provisioning and reconsideration of institutionalized practices. Each of the panelists is actively engaged in an e-health program, and drawing 
on their experiences, they consider the practical and research challenges involved in developing systems for co-producing health care and health care delivery.

\subsection{Workshop Paper Contributions}

Turning finally to the workshop papers, we see that the research themes investigated in completed research and research-in-progress papers are evident in these emerging research projects, that is, conceptualizing and innovating with service, globalization of service work, and development of IT-enabled services in public service arenas. To conceptualize services, Germonprez and Hovorka posit the information services view as a shift from the defined and predetermined services to a user-enabled real-time production environment of ad hoc information systems. Carter, Takeda, and Truex suggest a novel theorization on services. They introduce a new process model as to how emergence arises and is manifest in organizational discourse, which they aim to illustrate by drawing on the discourse and narratives of IBM in transitioning to a service-oriented company. In the area of IT in public services, Miscione and Aanestad highlight the importance of free and open source software and organizational learning in public administrations of developing countries, using the case of a health information system being implemented in Kerala, India. Tan, Tan, and Teo develop a research model that identifies factors influencing a blogger's intention to participate in a commercial exchange, for example between buyers and sellers of a service. The last two workshop papers seek to contribute to the growing IT service management area. In their paper, Tong, $\mathrm{Xu}$, and Pan aim to understand how dynamic capabilities are developed to facilitate software process improvement implementation in a small scale service-oriented company. Finally, Cuthbert, Pennesi, and McFarlane use a case study approach to develop a service information model whereby service information requirements are extracted to support the delivery of a service to a customer.

\section{CONCLUDING REMARKS}

The papers in this volume provide a rich milieu of approaches and draw on a wide range of theories to examine IT and transformations toward a service economy across four main themes. This diversity in "letting a thousand flowers bloom" is a strength of our 8.2 community, which has a lot to offer in understanding the social and political aspects of Bryson et.al.'s (2004) service worlds. It is timely that we as researchers at the intersection of information systems and organizations actively voice our contributions to this emerging research area. Not only are the trends of growth toward a service economy evident, but this growth in demand for service activity across industries has led to recent attempts to develop a new field of Service Science to integrate and bring coherence across silos of fragmented knowledge in a multidisciplinary approach (Chesbrough and Spohrer 2006).

Our conference has started some way down this track toward a multidisciplinary view on services. The diversity of scholars and the range of papers accepted for the conference reflects a breadth beyond what might traditionally be expected from an IS perspective on services, such as web services and service-oriented architectures (Spohrer 
and Riecken 2006), and indeed even the usual 8.2 community. For example, we welcome the perspectives on policy and government from colleagues in the information schools as well as those suggested by researchers coming from the manufacturing and management tradition.

Going forward, there are many opportunities for study in these service worlds. We are in favor of letting a thousand flowers bloom, but we also believe there is great value in cultivating research developments as they evolve and taking stock of and, where possible, categorizing the emerging concepts, theories, and methodologies for advancing our knowledge at both micro and macro-levels of research on IT and services. In doing so, we believe that future research on services should be embarked upon through innovative multidisciplinary research with scholars in a number of other fields, including marketing, strategy, operations, and organization theory. This research journey has only just begun, but it is one filled with a future of exciting challenges and opportunities.

\section{References}

Bryson, J., Daniels, P., and Warf, B. 2004. Service Worlds: People, Organizations, Technologies, London: Routledge.

Chesbrough, H., and Spohrer, J. 2006. "A Research Manifesto for Services Science," Communications of the ACM (47:7), pp. 35-49.

Chiasson, M., and Davidson, E. 2005. "Taking Industry Seriously in Information Systems Research," MIS Quarterly (29:4), pp. 599-606.

Gersung, C., and Resengren, W. 1973. The Service Society, Cambridge, MA: Schenkman Publishing Company.

Gustafsson, A., and Johnson, M. 2003. Competing in a Service Economy: How to Create A Competitive Advantage through Service Development and Innovation, San Francisco: John Wiley and Sons, Inc.

Spohrer, J., and Riecken, D. 2006. "Services Science," Communications of the ACM (49:7), pp. 31-32.

Vargo, S. L., and Lusch, R. E. 2004. "Evolving to a New Dominant Logic for Marketing," Journal of Marketing (68), pp. 1-17.

Vargo, S. L., and Lusch, R. E. 2008. "Service-Dominant Logic: Continuing the Evolution," Journal of the Academy of Marketing Science (36:1), pp. 1-10. 